Aus dem Physiologischen Institut der Universität in Kiel.

\section{Klinische Anwendung der Gaskettenmethode zur Bestimmung der Blutreaktion.}

\section{Von Rudolf Höber.}

Eine kürzlich von Ylppö ${ }^{1}$ ) veröffentlichte Abhandlung über den Wasserstoffionengehalt des Blutes veranlaßt mich, das Ergebnis einiger Versuche mitzuteilen, die im Wintersemester 1914/15 ausgeführt wurden und die seither aus äußeren Gründen nicht ganz zum Abschluß gebracht werden konnten.

Ich habe in der vierten Auflage meiner „Physikalischen Chemie der Zelle und der Gewebe“ (1914) auf S. 186 darauf hingewiesen, daß die ursprünglich von mir $^{2}$ ) angegebene Gaskettenmethode zur Bestimmung der Blutreaktion bisher der Klinik nur wenig Dienste geleistet hat, um Reaktionsstörungen in Richtung zunehmender Azidität, also kurz „Azidosen" zu erkennen, und habe angegeben, auf welche Weise die Methode ergiebiger zu gestalten wäre. So wie das Verfahren heute gehandhabt wird, findet man selbst bei recht ausgesprochener Säureintoxikation im Blut normale oder fast normale $\mathrm{H}^{\cdot}$-Werte. Das liegt daran, daß im allgemeinen bei Ueberproduktion von organischen Säuren regulatorisch die $\mathrm{CO}_{2}$-Spannung im Blut durch die Atmung so lange herabgesetzt wird, bis organische Säuren + Kohlensäure zusammen die normale $\mathrm{H} \cdot \mathrm{K}$-Konzentration ergeben. ${ }^{3}$ ) Daher ist es auch gerechtfertigt, eine Methode zur Messung der Azidosis auf die Bestimmung der $\mathrm{CO}_{2}$.Tension za gründen, so wie es Morawitz ${ }^{4}$ ) vor zwei Jahren getan hat.

Der Weg, den ich vorschlug, um die Gaskettenmethode zu Azidosisbestimmungen geeignet zu machen, war der, daß man erst die Kohlensäure aus dem Blut austreibt und danach die $\mathrm{H}$ - Konzentration im entgasten Blut bestimmt, und ich habe mich dann selbst davon überzeugt, daß dies Verfahren zum Ziel führt. Die Mitteilung meiner Ergebnisse ist nun durch die Veröffentlichung von Ylppö überholt.

Ylppös Mitteilungen beziehen sich auf den $\mathrm{H} \cdot$-Gehalt des Blutes von Säuglingen.

Er bestimmt bei jeder Blutprobe 1. die „regulierte WasserstoffzahI“ (Hasselbach), d.h. den $\mathrm{H} \cdot$-Gehalt des unveränderten Blutes, und 2. die „Grundwasserstoffzahl“", d. h. den H'-Gehalt des entgasten Blutes. Die Differenz ergibt die „, $\mathrm{CO}_{2}$-Regulationsbreite des Blutes", welche Ylppö in ähnlicher Weise als Maß der Azidose verwendet, wie Mora. witz die $\mathrm{CO}_{2}$ Tensionen bei seinem tonometrischen Verfahren. Nach meiner Meinung kann man, wie weiterhin erörtert werden wird, in der

1) Zschr. f. Kdhlk. 14. 1916 S. $268 . \quad{ }^{2}{ }^{2}$ ) Pflüg. Arch. 81. 1900 S. 522 u. 99. 1903 S. $572 .-{ }^{3}$ ) Siehe besonders Hasselbalch, Bioch. Zschr. 38. 1912, 46. 1912 u. 49. 1913. - ${ }^{4}$ ) Morawitz u. Walker, Bioch. Zschr. 60. 1914 S. 395.
Klinik von der ersten Messung absehen und sich auf die eine Bestimmung ఓm entgasten Blut beschränken.

Ich verfahre folgendermaßen:

5-10 ccm Blut, das durch Oxalat oder Hirudin ungerinnbar gemacht worden ist, wird in ein Tonometer (s. Fig.) von etwa $350 \mathrm{ccm}$ Inhalt gebracht. Bei a ist durch Druck. schlauch eine mit angefeuchteten Bimstein-

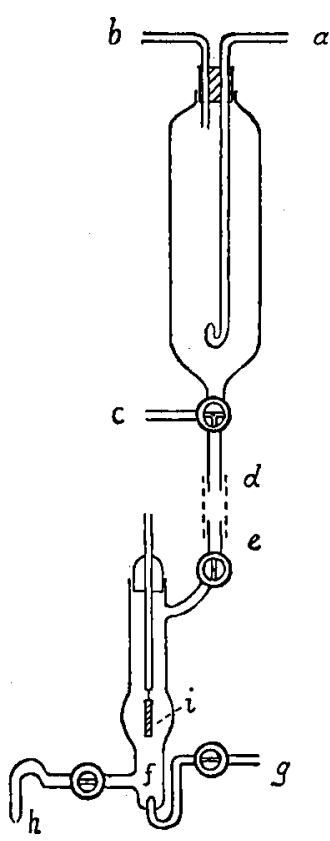
stücken angefüllte Flasche angeschlossen, ebenso bei $b$ eine zweite Flasche. Die Rohrstücke $\mathrm{c}$ und $\mathrm{d}$ sind ebenso wie die Bohrung des zwischen $c$ und $d$ liegenden Dreiweghahns mit Quecksilber verschlossen. Das Tonometer und die beiden Flaschen werden in ein Wasserbad von $38^{\circ}$ versenkt und mit einer an die Flasche 2 ange. schlossenen Wasserstrahlpumpe 15 Minuten lang evakuiert. Darauf wird durch die zweite Flasche hindurch unter Schütteln des Tono. meters Wasserstoff eingeleitet und abermals 10 Minuten evakuiert. Schließlich wird nochmals Wasserstoff eingeleitet, das Tonometer aus dem Wasserbad herausgehoben und bei d mit dem Ansatz e der Gaselektrode durch Schlauch verbunden, nachdem das Quecksilber aus $c$ und $d$ und dem Dreiweghahn entfernt worden ist. Bevor darauf das Blut aus dem Tonometer in das etwa $2 \mathrm{ccm}$ fassende Gefäß f der Elektrode übergeleitet wird, wird von $g$ aus unter sukzessivem Oeffnen der Hähne bei h und e längere Zeit Wasserstoff durch den Elektrodenraum und durch $d$ und $c$ geleitet. Danach erfolgt die Fïllung mit Blut so weit, daß der untere Rand des Platins i eben benetzt wird, und bis zur Oefinung bei h. Die Elektrode hat alsdann meist binnen einer Viertelstunde konstantes Potential; anderenfalls kann von $g$ aus nochmals Wasserstoff eingeleitet und bei e durch $d$ und $c$ herausgeleitet werden.

Auf diese Weise wurden die in der folgenden Tabelle verzeichneten Werte festgestellt.

Unter $\mathrm{p}_{\mathrm{H}}$ sind die Wasserstoffexponenten nach Soerensen, d.h. die negativen Logarithmen der $\mathrm{H} \cdot-$ Konzentrationen, unter $\left(\mathrm{H}^{\cdot}\right)$ die $\mathrm{H} \cdot$ Konzentrationen selbst angegeben.

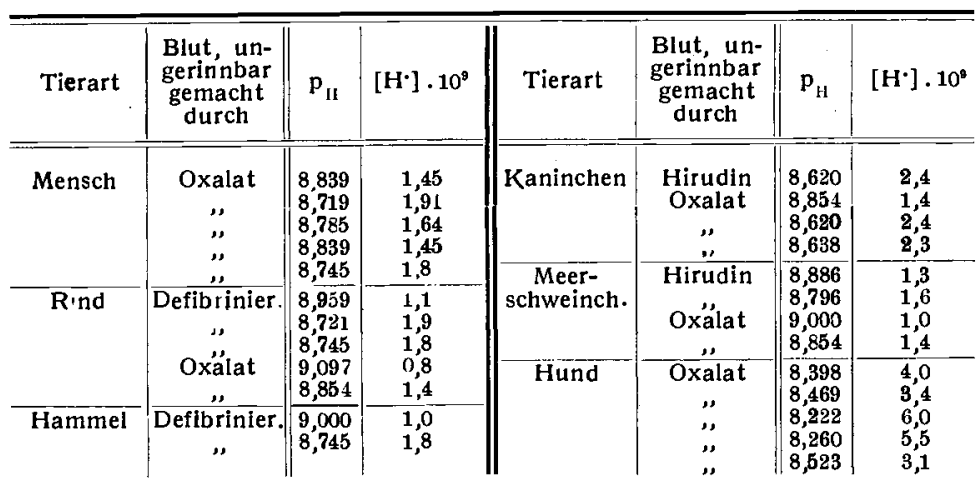

Die Tabelle lehrt, da $\beta$, wie ich schon vor 17 Jahren in meiner ersten Mitteilung ${ }^{1}$ ) über die $\mathrm{H}^{\cdot}-$ Konzentration des Blutes angegeben habe, das entgaste Blut ganz schwach alkalisch reagiert, und zwar ist offenbar die Reaktion dieses, geradeso wie die des unveränderten Blutes, einigermaßen konstant, sie schwankt bei Mensch, Rind, Hammel, Kaninchen und Meerschweinchen etwa zwischen $1,10^{-9}$ und $2,10^{-9}\left[\mathrm{H}^{\cdot}\right]$, nur beim Hund scheint der Wert etwas höher zu liegen.

Diesen Normalwerten habe ich einige Werte an die Seite zu stellen, welche für pathologisches Blut gelten und welche, ebenso wie die Angaben von Ylppö, lehren, daß a m entgasten Blut eine Azidose elektrometrisch nach$z u w e i s e n$ ist, wo die Messung a $m$ unveränderten Blut resultatlos bleibt.

1. Diabetes. Der Wasserstoffexponent für normales unverändertes, venöses menschliches Blut bei Zimmertemperatur beträgt im Mittel 7,56. Den gleichen Wert $(7,51-7,61)$ gibt

1) l. e. 
Michaelis ${ }^{1}$ ) für unverändertes diabetisches Blut nach Messungen von $\mathrm{Masel}^{2}$ ) an. Am entgasten Diabetikerblut fand ich folgende Werte:

\begin{tabular}{c|c|c|c}
\hline & $\mathrm{p}_{\mathrm{H}}$ & $10^{\circ}[\mathrm{H}]$ & \multicolumn{1}{c}{ B e m e r ku nge n } \\
\hline \hline 1. & 8,585 & 2,6 & $\begin{array}{l}\text { 1400 ccm Harn, } 1,9 \mathrm{~g}=0,137 \% \text { Azeton. } \\
\text { Azetessigsäure }+ \\
2500 \mathrm{ccm} \text { Harn, } 4,19 \mathrm{~g}=0,167 \% \\
\text { Azetessigsäure }+\end{array}$ \\
8. & 8,287 & 5,8 & $\begin{array}{l}3200 \mathrm{ccm} \text { Azeton. } \\
\text { Azetessigsän, } 3,8 \mathrm{~g}=0,119 \% \text { Azeton. } \\
\text { Azeton. }\end{array}$
\end{tabular}

Alle vier Messungen ergeben also Azidose, d. h. erhöhte Azidität im Blut bei Diabetes im Verhältnis zum Normalblut, bei welchem (s. Tabelle) für $10^{9}$. [H· $] 1,45-1,91$ gefunden wurde.

2. Strychninkrämpfe. Bei zwei mit Urethan tief narkotisierten Kaninchen wurde durch intravenöse Injektion einer 0,1\%igen Strychninlösung Tetanus erzeugt. Sofort nach Ausbruch der Krämpfe wurde künstliche Atmung eingeleitet. Vor und während der Krämpfe wurden Blutproben entnommen.

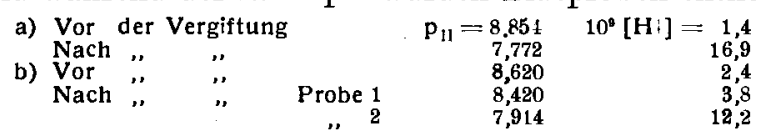

3. Urämie. Bei zwei Hunden wurden die Ureteren unterbunden; nach drei Tagen, während die Tiere gehungert hatten, bestand große Mattigkeit, aber kein Koma, kein Erbrechen, keine Krämpfe. Vor der Operation und drei Tage danach wurde Blut entnommen.

$$
\begin{array}{lrr}
\text { a) Vorher } & \mathrm{p}_{11}=8,488 & 10^{\circ}[\mathrm{H} \cdot]=\mathbf{3 , 2 5} \\
\text { Nachher } & 8,180 & 6,6 \\
\text { b) Vorher } & 8,509 & 3,1 \\
\text { Nachher } & 8,125 & 7,5
\end{array}
$$

Sowohl nach Strychninkrämpfen, als auch nach Ureterenunterbindung war also Azidose im Blut nachzuweisen.

Ich komme nun noch mit ein paar Worten auf die Messungen von Ylppö zurück, um auch an ihnen zu zeigen, daß meines Erachtens die $H^{\cdot}$-Messung am entgasten Blut im allgemeinen für die Klinik genügen wird und die Messung am unveränderten Blut entbehrlich ist.

Den Angaben von Ylppö entnehme ich folgende Werte, welche den Einfluß des Hungers auf die Beschaffenheit des

\begin{tabular}{|c|c|c|c|c|}
\hline & & $\begin{array}{c}\text { Regulierte } \\
\text { Wasserstoff- } \\
\text { zahl }\end{array}$ & $\begin{array}{c}\text { Orund- } \\
\text { Wasserstoff- } \\
\text { zahl }\end{array}$ & $\underset{\text { Regulations- }}{\mathrm{CO}_{2}-}$ \\
\hline $\begin{array}{rr}\text { Fall } & 60 \\
. & 61 \\
, & 62 \\
. & 63\end{array}$ & $\begin{array}{l}\text { vorher } \\
\text { nach } 40 \mathrm{Std} \text {. Hunger } \\
\text { verher } \\
\text { nach } 40 \mathrm{Std} \text {. Hunger } \\
\text { vorher } \\
\text { nach } 2^{1 / 2} \mathrm{Tg} \text {. Hunger } \\
\text { vorher } \\
\text { nach } 2^{1 / 2} \mathrm{Tg} \text {. Hunger }\end{array}$ & $\begin{array}{l}7,57 \\
7,59 \\
7,58 \\
7,62 \\
7,50 \\
7,41 \\
7,53 \\
7,53\end{array}$ & $\begin{array}{l}8,27 \\
8,09 \\
8,30 \\
8,23 \\
8,45 \\
8,04 \\
8,40 \\
7,98\end{array}$ & $\begin{array}{l}0,70 \\
0,50 \\
0,72 \\
0,61 \\
0,95 \\
0,63 \\
0,87 \\
0,45\end{array}$ \\
\hline
\end{tabular}
Blutes von Säuglingen darlegen (zur Terminologie s. S. 551).

Noch demonstrativer sind die Werte für Fälle von nicht

\begin{tabular}{|c|c|c|c|c|}
\hline & & $\begin{array}{c}\text { Regulierte } \\
\text { Wasserst off- } \\
\text { zahl }\end{array}$ & $\begin{array}{c}\text { Grund- } \\
\text { Wasserstoff- } \\
\text { zahl }\end{array}$ & $\underset{\substack{\mathrm{CO}_{2-} \\
\text { breite }}}{\mathrm{CO}_{2}-}$ \\
\hline Fall 74 & $\begin{array}{c}- \\
\text { Besserung } \\
\text { ", }\end{array}$ & $\begin{array}{l}7,41 \\
7,57 \\
7,49 \\
7,61\end{array}$ & $\begin{array}{l}7,86 \\
8,48 \\
8,36 \\
8,50\end{array}$ & $\begin{array}{l}0,55 \\
0,91 \\
0,91 \\
0,89\end{array}$ \\
\hline Fall 75 & Besserung & $\begin{array}{l}7,41 \\
7,44 \\
7,36\end{array}$ & $\begin{array}{l}7,90 \\
8.29 \\
8,26\end{array}$ & $\begin{array}{l}0,46 \\
0,85 \\
0,90\end{array}$ \\
\hline Falh 84 & Besserung & $\begin{array}{l}7,66 \\
7,45\end{array}$ & $\begin{array}{l}7,98 \\
8, .2\end{array}$ & $\begin{array}{l}0,32 \\
0,57\end{array}$ \\
\hline
\end{tabular}
letal verlaufender Säuglingsintoxikation:

Beide Tabellen lehren, daß die pathologischen Verhältnisse die Grund-Wasserstoffzahl verändern, während die regulierte Wasserstoffzahl im allgemeinen unbeeinflußt bleibt.

Schluß. Die Gaskettenmethode ist ein verhältnismäßig einfaches Verfahren, um eine Azidose des

1) Die Wasserstoffionenkonzentration, Berlin 1914 S. 105.

2) Zschr. f. kl. Med. 79. 1913 S. 1.
Blutes nachzuweisen, wenn man die Bestimmung am entgasten, d. h. $\mathrm{CO}_{2}$-freien Blut ausführt. 